\title{
ALGUNAS CONSIDERACIONES ACERCA DE LA CORRESPONDENCIA ENTRE MARTIN HEIDEGGER Y BERNHARD WELTE
}

\author{
César Lambert \\ Universidad Católica del Maule \\ clambert@ucm.cl
}

\begin{abstract}
Resumen
Este artículo examina la correspondencia entre Martin Heidegger y el teólogo cristiano Bernhard Welte, correspondencia publicada en 2003. Se pregunta por la relación intelectual que Heidegger tuvo con Welte. Distinguimos tres niveles: el interés de Heidegger por la Teología; la lectura, por parte de Heidegger, de algunos escritos de Welte; y un caso de discusión concerniente a la interpretación de autores de la tradición filosófica y su lugar en la historia de la filosofía.
\end{abstract}

Palabras Clave: Martin Heidegger, Bernhard Welte.

\section{Abstract}

This article analizes the correspondence between Martin Heidegger and the Christian theologian Bernhard Welte, published in 2003. We inquire into the intelectual relation Heidegger had with Welte. Three levels are distinguished: Heidegger's interest in Theology; Heidegger's reading of some Welte's texts; and one case of discussion regarding the interpretation of philosophers and their place in the history of philosophy.

Keywords: Martin Heidegger, Bernhard Welte.

$\overline{\mathrm{RA}}$

En 2003 se publicaron las cartas que, entre 1945 y 1976, intercambiaron el filósofo Martin Heidegger y su coterráneo, el teólogo católico Bernhard Welte. Este último es, ante todo, conocido por haber sido él quien hizo, a petición del propio Heidegger, el discurso fúnebre del filósofo en Messkirch (Welte 2003).

La relación entre ambos se articula desde aspectos de diferente índole: ambos son oriundos del pueblo de Messkirch; sus madres se conocían y apreciaban (Heidegger-Welte 2003, p. 6); a uno y otro los une la figura de Konrad Gröber, arzobispo de Friburgo entre 1932 y 1948, de quien Welte fue secretario; Heidegger y Welte tienen, cada uno a su modo, una estrecha relación con la Universidad de Friburgo, de la cual ambos fueron -aunque en épocas claramente distintas- rectores: Heidegger en 1933-34; Welte en 1955-56 (Silva Soler 2000, p. 21). Ambos vivieron la mayor parte de sus vidas en la ciudad de Friburgo, y allí murieron. 
Desde el punto de vista intelectual, la relación entre ellos es, a todas luces, asimétrica. Pues, Heidegger es una figura de talla mundial, ampliamente conocida, mientras que Welte es, principalmente, un profesor de Filosofía de la Religión, que, en general, solo es estudiado en pequeños círculos, y muchas veces a partir de Heidegger.

Por otra parte, en la obra de Welte se pueden constatar innegables influencias de Heidegger, que el propio teólogo, explícitamente, reconoce (Welte 2006, p. 17; Heidegger-Welte 2003, p. 26). En Heidegger, hasta donde yo entiendo, no hay una presencia de Welte. Lo que sí hay en él es un determinado interés por el futuro de la Teología y, como veremos aguas abajo, del pensar especulativo.

Así pues, lo que a propósito de ese contexto queremos aquí poner en consideración es la manera en que Heidegger entiende su relación intelectual con Welte. Ello a partir de las 21 cartas que le envía a Welte. No se trata, como propone un destacado intérprete heideggeriano, de preguntar por la relación de Heidegger con el cristianismo $(\text { Emad 2006, p. 198 })^{1}$-pregunta legítima e importante-, pero que quizá no sea la más exacta a efecto de comprender la relación Heidegger-Welte.

En las cartas de Heidegger se pueden distinguir tres niveles: [1] se constata un interés suyo por la Teología y por la Teología Fundamental; [2] Heidegger lee algunos textos de Welte y los examina con cierto detenimiento; y [3] a propósito de estos textos surge en Heidegger un caso de preguntas de interpretación respecto de autores de la tradición filosófica.

\section{Interés por la Teología}

Corren los días de mayo de 1946 y Martin Heidegger se encuentra internado en el sanatorio de Viktor von Gebsattel, en Badenweiler, desde donde le escribe a Welte a propósito de la muerte de la madre de éste. Cito tres párrafos de la carta del 12.5.1946:

Con gran interés leí parte de vuestro trabajo. Algunas tardes hemos concentrado nuestros diálogos de mesa en las preguntas de la Teología Fundamental. Ahora sólo le deseo una cosa: que usted llegue a la docencia lo más pronto posible, la cual es sana no sólo para los oyentes, sino para uno mismo.

Recientemente conocí también el libro de Karl Rahner Oyente de la Palabra [Hörer des Wortes]. Es peculiarmente dialéctico y no tan exitoso en la discusión

1 Allí, en lo fundamental sostiene lo siguiente: "[...] in what follows I shall take a look at this volume with two questions as my prime concern: (1) how did Heidegger himself perceive his relationship to Christianity and (2) how did Welte understand Heidegger's thought? By putting these two questions in the forefront of the discussion, I do not intend to pose Heidegger's own perception of his relationship to Christianity as a 'principle' against which Welte's understanding of Heidegger's thought should be measured. For Heidegger's perception of his relationship to Christianity, and Welte's understanding of Heidegger's thought are two differente matters" (Emad, 198). 
con Hegel. En el planteamiento fundamental hermoso, pero todavía muy poco sencillo, muy poco directo desde el asunto, lo que precisamente hace tan valiosa vuestra exposición.

Espero mucho que pronto haya otra vez una oportunidad para un diálogo (Heidegger 2006, p. 84) ${ }^{2}$.

¿Qué se constata en estos párrafos? Heidegger participa en las conversaciones de sobremesa, uno de cuyos tópicos es -según relata- la Teología Fundamental, vale decir, aquella disciplina de la Teología que lleva a cabo una reflexión acerca de las determinaciones básicas del cristianismo en sus aspectos formales, materiales, estructurales y existenciales (Seckler 1995, p. 227).

En ese contexto se inscribe la lectura de la tesis de habilitación de Welte, titulada La fe filosófica en Karl Jaspers y la posibilidad de su interpretación por medio de la filosofía tomista, terminada en 1946, pero publicada recién en 1949. Se entiende, entonces, que Heidegger sitúa la indagación weltiana en el marco de la Teología Fundamental, y que, dentro de ese marco, la propuesta es muy valiosa para él. Y es que Heidegger pone en paralelo la habilitación de Welte con la conocida obra de Karl Rahner Oyente de la Palabra (1941), que, siendo una fundamentación de la Filosofía de la religión o de Filosofía cristiana, es, a su modo, un tratado de Teología Fundamental (Rahner 1997, pp. 34-42, páginas pares).

Por su parte, el último de los párrafos citados de la carta hace patente que, más allá de las buenas maneras, a Heidegger le interesa retomar las conversaciones que, a tal respecto, ha tenido ya con Welte.

Pasemos al examen de otro fragmento epistolar. En 1964, Heidegger ha cumplido 75 años y Welte le envía un ejemplar del artículo "Die Gottesfrage im Denken Martin Heideggers" (Welte 1965, pp. 262-276) -del que nos ocuparemos, con más detalle, aguas abajo. Heidegger, a su vez, le escribe agradeciéndole el recuerdo. Así, en esta carta, con fecha del 13.10.1964, el filósofo hace referencia al diálogo de la Teología y la Filosofía. Cito:

Meticulosidad y juicio cuidadoso caracterizan también su artículo en memoria de G. Siewerth, por cuyo trabajo aquí le doy tardíamente las gracias. El diálogo entre la Teología y la Filosofía llega pues poco a poco a una dimensión fructífera y objetiva. Permanece vivo el deseo de que en la joven generación se despierte el pensar especulativo y ante todo que se ejercite estrictamente. En comparación con la época de hace 40 años ahora mucho se halla listo y abierto. Pero este alivio y la actual facilidad en el enunciar es a la vez un gran peligro. Éste sólo puede ser enfrentado en la cuidadosa educación para atenerse a lo concreto de las cosas [Sachlichkeit] (Heidegger 2006, p. 91) ${ }^{3}$.

2 La traducción fue levemente modificada por nosotros. El original se encuentra en: HeideggerWelte, 2003, p. 12.

3 La traducción fue levemente modificada por nosotros. El original se encuentra en: HeideggerWelte, 2003, pp. 19-20 
La afirmación de Heidegger según la cual el diálogo entre Filosofía y Teología llega, poco a poco, a una dimensión fructífera sigue, de modo inmediato, al comentario que le hace a Welte sobre el artículo que éste le había enviado. Hay que entender, entonces, que esa afirmación se refiere al contenido del texto: que allí acontece un diálogo entre ambas disciplinas, y que ese diálogo no constituye, por así decir, un continuo malentendido, antes bien, va siendo, cada vez más, verdadero y efectivo diálogo. Y eso es así en la medida en que tiende a lo que Heidegger denomina aquí "eine fruchtbare sachliche Dimension" (Heidegger-Welte 2003, p. 20), vale decir, una fructífera dimensión de carácter concreto. Esa dimensión viene a constituir, entonces, un campo común para Teología y Filosofía. Es lo que, en la cita, Heidegger llama el pensar especulativo, y que -no nos podemos abocar aquí a esa temática-ve en peligro en comparación con lo que pasaba en los años 20. Esencial es, según Heidegger, que este pensar especulativo, que incluye Filosofía y Teología, puede ser educado para que se atenga a lo concreto de las cosas. El trabajo intelectual de Welte realiza, pues, según Heidegger, el diálogo de esos saberes especulativos. Es posible, entonces, examinar, como veremos más abajo, el artículo "Die Gottesfrage im Denken Martin Heideggers" para precisar el tipo de pensar que ahí se pone por obra.

Un año y medio después, Heidegger le escribe al teólogo alemán con ocasión de su sexagésimo cumpleaños. Lo recuerda como muchacho sentado en el coro de la iglesia de San Martín, en Messkirch. En relación con la actividad de Welte, habla con simpatía de ella:

Usted ha logrado en una larga preparación una nueva forma de la docencia en Teología, que permite una discusión positiva con la época contemporánea y a la vez mantiene despierta la mirada a las grandes figuras en la tradición del pensar teológico. De esta forma se puede salvar del mejor modo al espíritu especulativo en contra del embate de formas nuevas y menos masivas del positivismo (Heidegger 2006, p. 93).

Aparece, nuevamente, la alusión al pensar especulativo; esta vez usa Heidegger la expresión "espíritu especulativo", que opone a las nuevas formas de positivismo. Por su parte, y a propósito del camino que Welte ha recorrido, Heidegger pone el acento en la larga preparación de su coterráneo en vistas a una nueva forma de actividad docente en Teología. No cabe duda, por el tono de las palabras, que hay valoración, estima, por ese estilo de labor teológica, particularmente por el hecho de que ese pensar no se ha encapsulado en sus propias reflexiones, sino que se ha atrevido a entrar en discusión con la época contemporánea, pero sin por ello desentenderse de los grandes autores de la tradición.

El interés heideggeriano por la Teología que aquí se constata apunta, antes que nada, a la dimensión común que es, precisamente, el espíritu especulativo. Asimismo, estima la actitud de discusión con la época contemporánea y el aspecto histórico de la disciplina. 


\section{Lectura de algunos textos de Welte}

Hemos mencionado ya que Heidegger lee algunos textos de Welte. No es éste un dato insignificante, pues aquél no se caracterizó, en general, por realizar una asidua lectura de sus contemporáneos. Es conocida la afirmación que hace en una carta, fechada el 18.09.32, a Elisabeth Blochmann: "Mientras tanto estudio mis manuscritos, es decir, me leo a mí mismo; y tengo que decir que, en lo positivo y negativo, es mucho más fructífero que otro tipo de lectura, respecto de la cual, por lo demás, tengo poco gusto y oportunidad" (Heidegger-Blochmann 1989, p. 53. Trad. nuestra).

En ese contexto, hay que reparar en el hecho de que Heidegger haya leído -por cierto, tres décadas después de la carta a Blochmann-algunos escritos de Welte: [1] Al menos parte de la tesis de habilitación de éste, La fe filosófica en Karl Jaspers y la posibilidad de su interpretación por medio de la filosofía tomista, de 1946 (HeideggerWelte 2003, p. 12). [2] El artículo, publicado por vez primera en 1964, "Die Gottesfrage im Denken Martin Heideggers" (Heidegger-Welte 2003, pp. 19-29; Welte 1965, pp. 262276). [3] El artículo "Thomas von Aquin und Heideggers Gedanke von der Seinsgeschichte", publicado en 1967 (Heidegger-Welte 2003, pp. 74-90). [4] El artículo "Gott im Denken Heideggers", que Welte le envía en 1974 (Heidegger-Welte 2003, p.35).

Hay que destacar, eso sí, que Heidegger lee solamente algunos textos de Welte: los que tienen que ver con su propio pensamiento, salvo el primero. No hay huellas en la correspondencia de que, por ejemplo, conociera el libro Auf der Spur des Ewigen, de 1965, que reúne una amplia gama de artículos. Tampoco hay testimonio de que conociera Heilsverständnis, de 1966, que constituye un tratado sistemático acerca de algunos supuestos para la comprensión del cristianismo -como reza el subtítulo de la obra-(Welte 2006, p. 15). Por último, tampoco hay reacción, por parte del filósofo, por el envío del libro Besinnung auf das Heilige (Casper-Hemmerle-Hünermann 1966), escrito en homenaje a Welte por tres de sus discípulos, y enviado a Heidegger el mismo año de su publicación (Heidegger-Welte 2003, p. 26).

Con todo, entre sumas y restas, hay, creemos, una preocupación de Heidegger que se constata en las lecturas arriba enumeradas, a saber: precisar la manera en que, a propósito de la pregunta por Dios, se lo está entendiendo en el marco de la Teología cristiana. Veamos, entonces, en concreto cada uno de los cuatro escritos.

En relación con el primero, la tesis de habilitación de Welte, ya hablamos aguas arriba, de manera que permítasenos ocuparnos, inmediatamente, del segundo, "Die Gottesfrage im Denken Martin Heideggers". Welte parte allí destacando el carácter de camino que tiene el pensamiento heideggeriano; camino que, por lo demás, ha pasado por diversas estaciones. Por eso, no hay que querer fijarlo, dice el teólogo alemán, en una posición determinada (Welte 1965, p. 262). En tal sentido, lo que Welte se propone aquí es "co-realizar el camino del pensar" de Heidegger (Welte 1965, p. 262).

Así, pone atención en lo que, en términos muy amplios, se da en llamar la pregunta por Dios (die Gottesfrage); y, para situarla, se atreve -nos dice-a poner en la mira la totalidad del camino del pensar de Heidegger (Welte 1965, p. 263). El punto 
de arranque de esta visión de conjunto lo constituye la noción de fenomenología, tal como es abordada en Ser y tiempo. El aporte de esta determinación heideggeriana es, según Welte, que hace patente la urgencia de poner a prueba el aparato conceptual tradicional de la Filosofía occidental; y hacerlo justamente desde lo que se muestra, sin distorsiones, desde sí mismo (Welte 1965, p. 265). Es el caso -continúa- de conceptos como los de sustancia, causa, fundamento, etc. "Al fin a al cabo, el concepto de Dios también pertenece a esos conceptos que tienen que ser revisados" (Welte 1965, p. 266. Trad. nuestra).

En relación con el concepto tradicional de Dios, el artículo que comentamos afirma que el destino del pensamiento occidental, marcado por el olvido del ser, tiene decisivas consecuencias para la pregunta por Dios, especialmente desde la época moderna (Welte 1965, p. 272). Él menciona dos.

La primera consecuencia es que Dios se tiene que convertir en un ente supremo, representado y asegurado por medio de demostraciones. Este ente supremo, es decir, Dios pensado metafísicamente, se convierte, a su vez, en el fundamento asegurador de todo ente. Dios es pensado como causa y -Welte cita a este efecto $L a$ constitución onto-teo-lógica de la metafísica- como causa sui (Welte 1965, p. 272). Este Dios, apunta Welte, no es según Heidegger un Dios divino, y cita un célebre pasaje del texto heideggeriano recién nombrado: "A este Dios, el ser humano no puede rezarle ni hacerle sacrificios. Ante la Causa sui el ser humano no puede caer temeroso de rodillas, así como tampoco puede tocar instrumentos ni bailar ante este Dios" (Heidegger 1990, p. 153) ${ }^{4}$.

Pero hay una consecuencia que, para Welte, es todavía más peligrosa: consiste en que Dios se torna un producto del pensamiento (ein Gemächte des Denkens). En tal sentido, el pensar subjetivo comienza a disponer de, y a determinar al ente supremo, esto es, al Dios de la metafísica (Welte 1965, p. 273). Desde esta perspectiva, la proclamación de la muerte de Dios por Nietzsche no es sino el efecto más extremo de la culminación de la metafísica occidental. Así, el Dios de la metafísica pierde toda fuerza edificadora, a tal punto que, aunque esté en la conciencia (Bewusstsein) de los seres humanos, ha dejado de presentarse como lo alto y sagrado. Es -dice Welte- lo que Heidegger llama, siguiendo a Hölderlin, la ausencia de Dios (Welte 1965, p. 273).

Welte intenta mostrar en este artículo que en Heidegger hay una pregunta por Dios; que el pensamiento heideggeriano permite hacerse cargo de la ausencia del Dios divino; y que, por tanto, este pensamiento filosófico hace posible re-pensar los conceptos fundamentales con que opera la Teología cristiana, en este caso, la noción de Dios como ente supremo.

En relación con la acogida, por parte de Heidegger, de este texto, permítasenos volver a citar su carta del 13.10.1964: "Meticulosidad y juicio ciudadoso caracterizan

4 La traducción fue levemente modificada por nosotros. El original se encuentra en: Heidegger, 2002, p. 64, así como en la propia versión bilingüe: Heidegger, 1990, p. 152. 
también su artículo en memoria de G. Siewerth, por cuyo trabajo aquí le doy tardíamente las gracias" (Heidegger 2006, p. 91).

El tercer escrito de Welte que Heidegger lee se titula, lo mencionamos arriba, "Thomas von Aquin und Heideggers Gedanke von der Seinsgeschichte" (1967). Tiene éste la peculiaridad de ser el único, en la correspondencia, donde Heidegger discute una tesis filosófica de Welte. Lo veremos más abajo.

El texto se articula de la siguiente forma: primero resume las reflexiones heideggerianas sobre la metafísica occidental; después se pregunta si Tomás de Aquino es parte de la metafísica así entendida, y para ello expone la doctrina tomista del ens y el esse; en tal contexto se concentra en el concepto de Dios como ipsum esse subsistens, y presenta el pensamiento de Meister Eckhart sobre Dios como consecuencia de la metafísica de Tomás de Aquino. La tesis defendida es que estamos, aquí, ante una incoada superación de la metafísica en el sentido de Heidegger.

Veamos con más detalle lo que Welte expone. Según él, Heidegger sostiene que, en la metafísica occidental, hay una dimensión no pensada, pero desde la cual lo explícitamente pensado en ella recibe su espacio esencial (Welte 2003, p. 74). Eso oculto es lo que Heidegger denomina el ser y su despejo; por eso, la metafísica está determinada por la ocultación de eso no pensado que es el ser. Por otra parte, el evento destinal del despejo del ser (das geschickliche Ereignis der Lichtung des Seins) produce, saca a luz, las distintas épocas del pensar (Welte 2003, p. 77). Desde este punto de arranque, Heidegger reflexiona acerca del pensamiento occidental en total, o sea, desde sus inicios griegos hasta su consumación en Nietzsche. Asimismo, según Welte, ha de repararse en lo que Heidegger denomina la superación de la metafísica. En efecto, ha llegado el momento -cuando el imperio de la metafísica llega a sus extremos en la técnica- de pensar lo no pensado allí, y, así, volver al destino inicial del ser (Welte 2003, p. 79).

Así pues, a propósito de la pregunta que Welte se hace, si el pensamiento de Tomás de Aquino pertenece a la metafísica como olvido del ser, lo que al teólogo alemán le importa es precisar la cercanía y diferencia de Santo Tomás respecto de Heidegger (Welte 2003, p. 80).

La exposición de Welte arranca de la conocida afirmación tomista según la cual aquello que el intelecto concibe primero como lo más conocido (notissimum) es el ente (Welte 2003, p. 80). Asimismo, sostiene que Tomás de Aquino distingue entre acto de ser y ente: "Distinguitur actus essendi ab eo, cui actus ille convenit" -como se dice en De Veritate, art. 1, ad 3 (Welte 2003, p. 81). Se trata, dice Welte, de la diferencia del ente respecto de su entidad, pero que no es la diferencia ontológica como la entiende Heidegger.

Ahora bien, en lo que concierne a la diferencia de actus essendi y ens Tomás de Aquino hace, según Welte, una diferenciación de tres casos: [1] el ser es la actualidad de todas las cosas. Es lo que hace que el ente sea, digamos, real; [2] en el ámbito de lo que concibe el intelecto tenemos el esse commune, vale decir, el ser en general, que se da, única y exclusivamente, en el intelecto que lo concibe, y [3] el ipsum esse subsistens, 
que constituye la forma metafísica, para Tomás de Aquino, de hablar de Dios (Welte 2003, pp. 82-83).

En los tres casos se trata de la entidad del ente, solo que en el tercero se da una dificultad: el que, en el ser infinito, el portador del ser se identifique con el ser que se le predica. "Dei essentia est suum esse", afirma a este respecto la Summa theologica (Welte 2003, pp. 83-84). ¿Constituye aquí el ser la entidad de ese ente? He ahí la pregunta de Welte. La respuesta tiene dos aspectos, en el sentido de que, por una parte, estamos ciertamente ante el primum ens o sustancia divina; pero, por otra parte, hay que poner en relación esa identidad con la afirmación de Tomás según la cual "Deus non [est] in aliquo genere", que se encuentra en Summa theologica I, q. 3 ad 5; y en Summa contra gentiles, I, c. 25 (Welte 2003, p. 84). Esta tesis tomista apunta, según Welte, a que Dios no es, en caso alguno, un ente; a Dios no se le predica el ser. Desde esta perspectiva, Dios no es un ente, y hemos abandonado el terreno de la entidad. "La afirmación, así entendida, trasciende el ámbito del ente y su entidad. Con ello remite más allá del ámbito de la metafísica en el sentido de Heidegger. La afirmación se encuentra en el límite de la metafísica" (Welte 2003, p. 85. Trad. nuestra).

Esta noción de Tomás de Aquino contiene, a modo de germen, la posibilidad -según Welte- de saltar fuera del ente en total y, así, superar el determinar conceptualizante en dirección a aquello que está allende todo concepto. La metafísica de Tomás contiene, pues, el germen de la posibilidad de la superación de la metafísica, pero - dice Welte- no era el tiempo para que ese germen se desarrollara (Welte 2003, p. 88).

En esa dirección, también entiende Welte el aporte de Meister Eckhart, cuyo pensamiento estaría vinculado a la afirmación "Deus non est in aliquo genere". De allí habría desplegado su noción de apartamiento (Abgeschiedenheit): ante lo que no se encuentra en el modo del ser y del afirmar, todo concepto, toda afirmación, debe caer, silenciarse. Por eso, para Eckhart, el Altísimo es experimentado como silencio, como nada, como desierto (Welte 2003, p. 89). Así, Eckhart y la superación de la metafísica que él lleva a cabo no son sino consecuencia, para Welte, de la metafísica de Tomás de Aquino (Welte 2003, p. 90). Veremos, aguas abajo, en qué sentido Heidegger no comparte esta visión y la discute con Welte.

Pasemos al examen del cuarto texto weltiano del que se tiene certeza que fue leído por Heidegger. Se trata del artículo "Gott im Denken Heideggers", cuya historia ha sido reconstruida por nosotros en otro lugar (Lambert 2006). El escrito parte, al igual que "Die Gottesfrage im Denken Martin Heideggers", del que constituye una reelaboración, según explica el propio autor (Welte 2003, p. 91), del concepto de fenomenología; en el enfoque fenomenológico se palpa una voluntad de superar el sujeto y la subjetividad; en relación con lo que se muestra, donde siempre cabe que eso se muestre de diversas maneras, Welte destaca que es propio de esos distintos modos de mostrarse que, a veces, ello se muestre como algo distinto de aquello que dicen los conceptos y las representaciones habituales (Welte 2003, pp. 92-93). Es el caso de lo que mienta la palabra Dios. 
Asimismo, respecto de Dios puede suceder que el juego de mostración y ocultamiento tenga un carácter diverso que en otros casos. "El mostrarse de lo que se muestra puede modificarse a tal punto que lo que se muestra se retenga y oculte, pero justo en este ocultamiento se anuncie o muestre. En ese caso el ocultamiento se convierte en una forma de mostrarse" (Welte 2003, p. 93. Trad. nuestra).

El texto aborda, acto seguido, el tratamiento heideggeriano de la nada y de la diferencia ontológica. En este contexto, Heidegger se pregunta qué es el ser, y en la Carta sobre el humanismo responde "Él 'es' él mismo" (Welte 2003, p. 97). Welte dice a este efecto que el ser se muestra justamente como ser, y ello implica que la palabra "ser" ha de permanecer en su carácter propio, sin que se la entienda desde conceptos habituales. Con todo, la afirmación "El ser es él mismo" no excluye la posibilidad de que el ser busque, por así decir, otros modos de mostrarse. Es posible que el ser busque otros modos de patentizarse. Junto con hacerse patente como nada -o como verdad- se podría mostrar aun de otras maneras. A este propósito, Welte cita una expresión de Heidegger que aparece en el Epílogo a ¿Qué es metafísica? Allí habla Heidegger de la misteriosa multivocidad de la nada (Welte 2003, p. 98). Pues bien, puesto que la fenomenalidad del ser no puede ser fijada de antemano y ella cambia históricamente, cabe la posibilidad de que el ser se muestre, alguna vez, como lo sagrado, lo divino (Welte 2003, p. 98).

Por otra parte, respecto del Dios de la metafísica, insiste Welte que no es Aquel ante quien el ser humano se postra y le reza; el Dios causa sui no es un Dios divino, sino producto del pensamiento (Welte 2003, p. 102). La consecuencia de que Dios sea pensado metafísicamente se percibe en los trazos fundamentales que determinan una época. Así, corresponde hablar, en la época del imperio de la técnica, de la ausencia de Dios (Fehl Gottes), ausencia que se extiende más y más (Welte 2003, p. 103). ¿Cómo entender esta ausencia de Dios? Welte afirma que se trata de "la urgencia del ocultamiento y [...] del no-manifestarse de la plenitud del epifánico Dios divino" (Welte 2003, p. 105. Trad. nuestra). Ahora bien, el pensamiento que supera la metafísica, afirma Welte, es capaz de entrever que el surgimiento del Dios divino, si es que alguna vez acontece, se llevará a cabo en la cercanía del ser. Y el pensamiento de Heidegger apunta precisamente a la preparación de la superación de la metafísica occidental, y ello también implica una preparación para la superación del Dios de la metafísica (Welte 2003, p. 105).

En este marco hace Welte referencia a la interpretación heideggeriana de Hölderlin: el despejo del ser comparece, cuando se lo piensa desde la cercanía de la palabra poética, como lo jovial (das Heitere), lo que trae la salvación (das Heile) y como lo sagrado (das Heilige). A su vez, lo sagrado ha de ser entendido como la huella hacia la divinidad. Pero aquí se da la paradoja de que lo sagrado se manifiesta y el Dios permanece lejos; en la noche de la nada se guarda y se oculta también el Dios; claro que éste aguarda aparecer a su debido tiempo (Welte 2003, p. 109).

Welte concluye el artículo con una referencia a las palabras que Heidegger dirige a la comunidad de Amriswil, en septiembre de 1969, con ocasión de su octogésimo cumpleaños (Heidegger 2000, pp. 715-717); palabras que Welte conocía en la 
versión del Neue Zürcher Zeitung. Heidegger pregunta en esa ocasión: "Ist unser Wohnen der Aufenthalt in einem Vorenthalt des Hohen?" (Welte 2003, p. 113; Heidegger 2000, p. 717), es decir: "¿Es nuestro habitar [el mundo] la permanencia en un retenerse de lo Alto?". Con esta última palabra, Heidegger hace referencia al Dios divino de Hölderlin, y pregunta si nuestro habitar el mundo no es, en el fondo, un mantenerse en la situación de que el Dios divino - vale decir, Aquel ante quien se postra el creyentese retrae y retiene en el ocultamiento. Asimismo, Welte plantea que ese interrogante de Heidegger pueda ser entendido como un resumen de todo el camino de su pensar (Welte 2003, p. 113).

Heidegger, por su parte, le escribe a Welte a propósito de este artículo. Por cierto, le agradece el envío y valora el estilo de pensar del teólogo:

Su co-pensar minucioso y cuidadoso es tan satisfactorio como extraordinario. Por lo general, se suele clasificar mis intentos precozmente en alguna categoría o aprovecharse de ellos de manera apologética (Heidegger 2006, p. 104) $)^{5}$.

También le hace un par de recomendaciones:

Usted sigue claramente una línea de mi pensar en sus estadios cambiantes. Podría enriquecerse, como usted mismo sabe, por ejemplo con una discusión sobre la esencia de la técnica moderna y sobre la -todavía no pensadafundación de la ciencia moderna dentro de esta esencia (Heidegger 2006, p. 105).

Por último, le hace una propuesta en vistas de la publicación del texto, que tiene que ver con el acento que ha de ponerse en el carácter de camino del pensar de Heidegger. Dice la carta:

No tengo dudas acerca de su texto, pero sí tengo una propuesta. En la publicación podría pedir al lector mediante una nota a pie en la primera página que considere primero el apartado final (p. 28). De esta manera se evitaría que, en contra de su propósito, alguien saque de su texto alguna tesis fija y opiniones incoherentes, en lugar de permanecer, de camino, en actitud de pregunta (Heidegger 2006, p. 105) ${ }^{6}$.

El apartado final al que hace referencia Heidegger corresponde, sin duda, a los dos últimos párrafos del texto de Welte. Allí se dice lo siguiente:

Hasta aquí se ha de seguir el camino del pensar. Nosotros lo hicimos en la medida que arriesgamos un intento de interpretación. El intento no está

5 La traducción fue levemente modificada por nosotros. El original se encuentra en: HeideggerWelte, 2003, p. 36.

6 La traducción fue levemente modificada por nosotros. El original se encuentra en: HeideggerWelte, 2003, p. 37. 
protegido de errores. En tanto que debía ser visto en él algo de lo que acontece en el pensamiento de Heidegger, se debe resumir esto de la siguiente manera: allí acontece escuchar y esperar. Acontece esto: entregarse confiadamente a orientaciones ocultas para aprehender, en la época del olvido del ser y del olvido de Dios, algo del oculto Dios divino en un terreno allende la metafísica y por una vía que ha dejado tras de sí, con la metafísica, también las habituales seguridades de la racionalidad.

Este camino del pensar que sigue las huellas de la divinidad que se retrae está siempre en peligro y es también peligroso. Pero en toda su cuestionabilidad da mucho que pensar (Welte 2003, p. 114. Trad. nuestra).

A Heidegger le interesa que un trabajo como el del teólogo cristiano no derive en una forma de apología que solamente se sirva, instrumentalmente, de su pensamiento. Pero, a la vez, vale la pena poner de relieve que la lectura weltiana pone la pregunta por Dios en un lugar central del pensar de Heidegger. Y esto no es, en caso alguno, rechazado por el filósofo.

\section{Discusión con Welte}

En este apartado queremos mostrar que, en un caso específico, Heidegger entra a discutir una tesis de Welte. No discute una tesis respecto de su propio pensamiento y la forma como Welte lo interpreta, sino la lectura que hace éste de la metafísica de Santo Tomás y de Meister Eckhart en el artículo "Thomas von Aquin und Heideggers Gedanke von der Seinsgeschichte".

Heidegger sostiene que no ve la relación que hace Welte entre las afirmaciones tomistas acerca de que Dios es el ser mismo subsistente y que Dios no se encuentra en género alguno (Heidegger-Welte 2003, p. 29). En efecto, la subsistentia es el primer $\gamma \varepsilon$ $v$ o $\varsigma$ de los genera; Heidegger remite aquí a Aristóteles (Metafísica B 3, 998b 22):

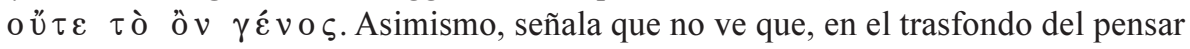
de Tomás de Aquino, esté lo que Welte ve en él. No así en el caso de Meister Eckhart y la afirmación de éste: "Sed etiam Deus quod Deo non convenit esse nec este ens, sed est aliquid altius ente". Lo que afirma Eckhart no se encuentra, dice Heidegger, en ninguna otra parte (Heidegger-Welte 2003, p. 29).

Sin embargo, a continuación reconoce no ser especialista en estas materias. Pero insiste: cree que lo que Welte le atribuye a Tomás de Aquino solo es obra de Eckhart (Heidegger-Welte 2003, p. 30).

Hay que reparar, en este punto, que es el único en toda la correspondencia donde explícitamente Heidegger se muestra en desacuerdo con una interpretación de Welte. Llama la atención que Heidegger tiene mucho cuidado con las afirmaciones que hace, pues reconoce no ser experto en Filosofía Medieval, pero la lectura weltiana de Tomás no logra, al parecer, convencerlo. La interpretación que el teólogo hace de su propio pensamiento, pese al innegable esfuerzo por encontrar allí aspectos teológicos o de Filosofía de la Religión, no le resulta, en principio, problemático. Al menos, no 
explícitamente. Sí, en cambio, la lectura weltiana que pretende ubicar a Tomás de Aquino en una corriente que anticipa la superación de la metafísica, tal como Heidegger la plantea.

\section{A modo de conclusión}

Hemos intentado precisar, en el marco de la correspondencia Heidegger-Welte, cuál es el enfoque que Heidegger tiene respecto de la obra del teólogo Bernhard Welte. Hemos distinguido a este efecto tres distintos niveles, y hecho ver en cada uno la actitud de Heidegger. Es cierto que la relación entre ambos no es, para nada, simétrica, pues Welte recepciona mucho de Heidegger, pero éste casi nada de aquél. Sin embargo, hay algunos elementos en Welte que a Heidegger le parecen de interés. Es lo que hemos querido mostrar en este artículo.

Asimismo, se constata que, aunque Welte tiene una cátedra ordinaria de Filosofía de la Religión (Christliche Religionsphilosophie) en la Facultad de Teología de la Universidad de Friburgo, y que en varios de sus escritos aparece en el subtítulo -como adjetivo- el término "filosófico" (Welte 1965; Welte 2006), Heidegger siempre entiende que está tratando con un teólogo, no con un filósofo. Ahí se da, al menos en ciernes, un desencuentro.

Por otra parte, lo que la investigación tiene aún que determinar a este respecto es: en qué grado conoció Heidegger la tesis de habilitación de Welte y otros escritos suyos más relevantes, como Heilsverständnis; en relación con otros pensadores cristianos, como Max Müller - cuya correspondencia con Heidegger también fue publicada recientemente (Heidegger 2006) - o Gustav Sieweth, ¿hay paralelos, divergencias, caminos comunes? Asimismo, visto desde el propio pensamiento de Welte, hay que determinar qué elementos del pensamiento de Heidegger recepcionó en mayor grado. Una pista es, vaya por caso, la noción de comprensión (Welte 2006, p. 17). En fin, hay una serie de preguntas por esclarecer con el propósito de reconstruir las relaciones intelectuales respecto de Heidegger en los círculos universitarios de Alemania y, especialmente, de Friburgo.

\section{Referencias bibliográficas}

Casper, Bernhard-Hemmerle, Klaus-Hünermann, Peter (1966), Besinnung auf das Heilige. Freiburg: Herder.

Emad, Parvis (2006), "Martin Heidegger -Bernhard Welte. Correspondence seen in the Context of Heidegger's Thought", en Heidegger Studies, vol. 22. Berlín: Duncker $\&$ Humblot.

Heidegger, Martin (1990), Identidad y diferencia. Identität und Differenz. Edición bilingüe, trad. de H. Cortés y A. Leyte. Barcelona: Anthropos. 
(2000), Gesamtausgabe. Band 16: Reden und andere Zeugnisse eines Lebensweges. Frankfurt a. M.: Vittorio Klostermann.

(2002), "Die onto-theo-logische Verfassung der Metaphysik", en Identität und Differenz. Stuttgart: Klett-Cotta, pp. 31-67.

(2006), Cartas a Max Müller y Bernhard Welte, traducción de Ángel Xolocotzi y Carlos Gutiérrez. México D.F.: Universidad Iberoamericana.

Heidegger, Martin-Blochmann, Elisabeth (1989), Briefwechsel 1918-1969. Marbach am Neckar: Deutsches Literaturarchiv.

Heidegger, Martin-Welte, Bernhard (2003), Briefe und Begegnungen. Stuttgart: KlettCotta.

Lambert, César (2006), "La pregunta por Dios en el pensamiento de Heidegger. Una aproximación desde la perspectiva de Bernhard Welte", en Herrera, María Fernanda-Brickle, Patricio-Lambert, César [ed.], Diálogo intercultural. Primer Encuentro Académico Chile-Bulgaria, Universidad Católica del Maule; New Bulgarian University, Sofia, pp. 97-124.

Rahner, Karl (1997), Sämtliche Werke, tomo 4: Hörer des Wortes. Schriften zur Religionsphilosophie und zur Grundlegung der Theologie. Freiburg: Herder.

Seckler, Max (1995), artículo "Fundamentaltheologie", en Lexikon für Theologie und Kirche. Ed. por Walter Kasper, tomo 4. Freiburg: Herder.

Silva Soler, Joaquín (2000), La verdadera religión. Un diálogo con Bernhard Welte. Universidad Católica del Maule, Colección Tabor n ${ }^{\circ} 18$, Talca.

Welte, Bernhard (1965), Auf der Spur des Ewigen. Philosophische Abhandlungen über verschiedene Gegenstände der Religion und der Theologie. Freiburg: Herder.

(2003), "Suchen und Finden. Ansprache zur Beisetzung Martin Heideggers am 28. Mai 1976", en Heidegger, Martin-Welte, Bernhard (2003), pp. 124-127.

(2006), "Heilsverständnis. Philosophische Untersuchungen einiger Voraussetzungen zum Verständnis des Christentums", en Bernhard Welte Gesammelte Schriften, tomo IV/1: Hermeneutik des Christlichen, editado por Bernhard Casper, Freiburg: Herder. 